\title{
El uso de sensores inerciales para el monitoreo de las sesiones de
}

\section{entrenamiento en cancha}

\author{
Cyril Genevois, Christel Amsallem, Cédric Brandli y Isabelle Rogowski
}

\section{RESUMEN}

Las innovaciones tecnológicas proporcionan mayor información a los entrenadores por medio de herramientas prácticas que les permiten tener mayor información sobre la actividad de los jugadores durante el entrenamiento y la competición. Este artículo presenta un estudio que utiliza los sensores inerciales, integrados en una muñequera, para cuantificar los diferentes tipos de tiros ejecutados por los jugadores durante una semana de entrenamiento pre-temporada, y compararlos con las exigencias de la competición.
Palabras clave: monitoreo del entrenamiento, dispositivo conectado, prevención de lesiones

Recibido: 27 Junio 2018

Aceptado: 10 Julio 2018

Autor correspondiente:

Cyril Genevois.

Correo electrónico:

Cyril.genevois@aol.fr

\section{INTRODUCCIÓN}

El manejo de la carga de entrenamiento y su relación con el riesgo de lesiones constituyen un punto de interés importante para los científicos del deporte en estos últimos años (Soligard y cols, 2016). Más específicamente en el tenis, se afirma que no se trata de la carga en sí, sino de una transición inapropiada a una carga mayor, llamada "el camino hacia la carga" que produce lesiones (Pluim y Drew, 2016; Rogowski y cols., 2016). Se demostrado recientemente que las lesiones en la parte superior del brazo, y la frecuencia de tratamientos en el torneo, han aumentado $\geq 2.4$ veces en ambos sexos en el Grand Slam del Abierto de Australia en un período de 5 años (Gesheit y cols., 2017). Estas lesiones son el resultado directo de las cargas mecánicas impuestas sobre el sistema musculo-esquelético (especialmente durante el servicio), y es conveniente considerar algunas medidas sobre el golpeo de la pelota en la exposición del miembro superior/ cuerpo. Además, los estudios demuestran ciertas diferencias entre los jugadores junior y senior en la cantidad de golpes durante los partidos, algo que los entrenadores deben considerar al planificar las sesiones de entrenamiento para cumplir con las exigencias de la competición (Myers y cols., 2016; Kovalchik y cols., 2017; Perri y cols., 2018). Para cuantificar la cantidad de tiros, los entrenadores pueden utilizar sensores inerciales que son no invasivos, son portátiles y discriminan entre los golpes de tenis (Whiteside y cols., 2017).
El objetivo de este estudio es cuantificar la cantidad y la intensidad de los golpes (tasa de golpes por minuto) ejecutados por los jugadores junior masculinos durante sus sesiones en cancha utilizando los sensores inerciales durante una semana. Esa semana de entrenamiento se llevó a cabo durante la pretemporada, apuntando a preparar a los jugadores, específicamente para los futuros torneos; el subsiguiente análisis de datos se utilizó para proporcionar la información a los entrenadores sobre la potencial brecha entre el contenido de las sesiones en cancha y las exigencias de la competición.

\section{MÉTODO}

Datos de cinco sesiones de tenis en cancha, jugadores junior de 14 años (edad: $15.4 \pm 2.0$ años, entre los 13 y los 19 años de edad, altura: $172.8 \pm 9.9 \mathrm{~cm}$, peso: $60.0 \pm 10.2 \mathrm{~kg}$, años de experiencia: $9.7 \pm 3.1$ años, entrenamiento semanal: $12.0 \pm 2.5$ horas, Número Internacional de Tenis $($ ITN) = 3) se analizaron utilizando una muñequera inteligente con sensor integrado en la mano dominante (Babolat Pop) (Figura1). La actividad del jugador se rastreó durante la sesión de tenis, y la información se envió de manera inalámbrica a un dispositivo móvil para ser desglosada golpe a golpe. 


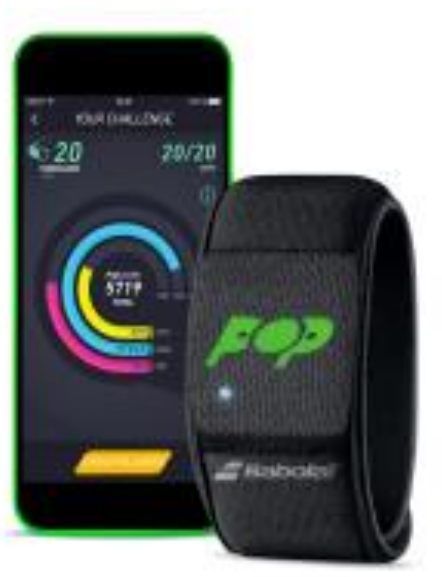

Figura 1. Dispositivo Babolat Pro.

Se calculó el número total de tiros y el número total de tiros por minuto para todo el grupo. El análisis descriptivo incluyó la desviación estándar y promedio para los servicios, golpes de derecha y de revés para las cinco sesiones. Se calculó también la correlación entre la edad y el número de tiros. Finalmente, se compararon los resultados entre los tipos de golpe en la misma sesión, utilizando las pruebas-t para alumnos, para muestras de pares con fijado en 0.05 . Se realizaron todos los análisis estadísticos utilizando el programa SPSS 11.0 (SPSS, Inc., Chicago, IL, USA)

\section{RESULTADOS}

La Figura 2 muestra la distribución promedio de los golpes de derecha, revés y servicios golpeados durante cada una de las cinco sesiones de tenis para todo el grupo.

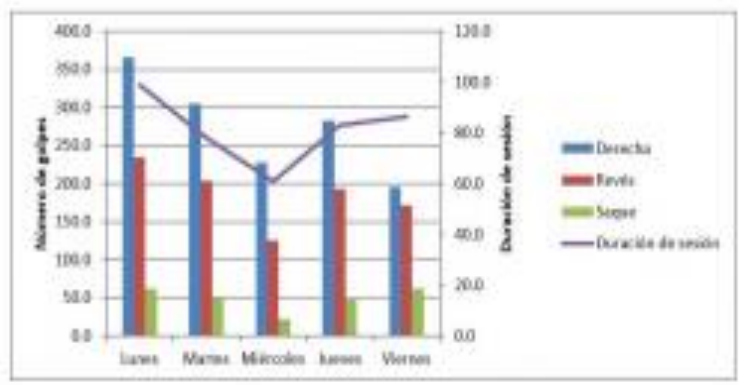

igura 2. Distribución relativa promedio de los tiros de tenis durante la emana de entrenamiento.

Como promedio, la duración de una sesión de tenis fue de 87.0 \pm 32.3 minutos, en los cuales los jugadores golpearon $291.1 \pm$ 150.5 golpes de derecha, $198.1 \pm 100.6$ golpes de revés, y 53.5 \pm 33.7 servicios. El número semanal promedio de golpes de derecha fue significativamente mayor que el de golpes de revés $(p<0.05)$. Tanto el número promedio semanal de golpes de derecha y revés fueron ambos significativamente mayores que el de golpes de servicios $(p<0.05)$. No hubo correlación entre la edad y el número de tiros golpeados durante las cinco sesiones de entrenamiento.

\section{COMENTARIO}

El principal resultado muestra una gran disparidad entre el promedio de servicios, golpes de derecha y de revés ejecutados en cada sesión. La relación promedio golpe de derecha/golpe de revés en nuestro estudio es $1.58 \pm 0.64$, superior a $1.24 \pm 0.37$ encontrado para los jugadores profesionales masculinos en competición (Reid y cols, 2016). Si bien el juego moderno parece caracterizarse por el exagerado número de golpes de derecha, no debe dejarse de lado la mejora de los golpes de revés. Obviamente, un estudio reveló que los golpes de derecha se asocian con una mayor cantidad de puntos ganados, mientras que se pierden más puntos con el revés cuando es jugado como tiro final (Cam y cols., 2013). Se podría decir que estos resultados no son sorprendentes si un tiro se juega (o se practica) más que el otro. Además, la carga de entrenamiento externa promedio parece no cumplir con las exigencias de la competición, que puede ser el objetivo de la pretemporada. Las intensidades (golpes/min) de los golpes de fondo varían de 4,3 $\pm 0,6$ a 6,8 $\pm 1,6$ y son inferiores a las observadas por Murphy y cols. (2016) para las sesiones de entrenamiento $(7 \pm 1.0)$, el juego de partidos simulados (10 \pm 5.1) y los torneos $(14 \pm 3.6)$. La diferencia podría deberse al mayor tiempo de descanso y/o más foco técnico/ táctico.

Con respecto al número de servicios, fue inferior a los 120 servicios propuestos por Myers y cols. (2016). Nuestros resultados se asemejan a los de Perry y cols. (2018) quienes observaron que el número de servicios durante una sesión de entrenamiento era significativamente inferior al de la competición para los jugadores masculinos menores de 15 años ( $38.6 \pm 24.2$ vs $82.0 \pm 24.8$ ). Como las agendas de torneos de los juniors suelen ser condensadas, los jugadores deben jugar varios partidos en pocos días, con un total de servicios que excede el de su semana de entrenamiento. Esta diferencia de volúmenes de servicios en la competición, comparada con el entrenamiento, sugiere que los entrenadores deben planificar mejor las cargas de entrenamiento de saque (volumen e intensidad) para coincidir con la competencia y asegurar reducir el riesgo de lesiones por exposición inadecuada. Durante las sesiones de entrenamiento se pueden implementar diferentes recomendaciones para mejorar la eficiencia del servicio y disminuir el riesgo de lesiones por sobrecarga del hombro. En primer lugar, el volumen y la intensidad de los servicios deben variar de una sesión a otra, para permitir la regeneración del tejido y deben planificarse con intervalos simulando el juego real (Myers y cols., 2016). En segundo lugar, otras modalidades de entrenamiento, como las imágenes motrices (Guillot y cols, 2012), o el entrenamiento físico (Fernández-Fernández y cols, 2013), han demostrado su efectividad a la hora de mejorar el rendimiento en el servicio con los jugadores juniors, y se pueden combinar con un volumen de servicio menor. Finalmente, es también 
importante limitar los desequilibrios de fuerza y del rango de movimiento entre los rotadores internos y externos, siguiendo un programa regular de prevención de lesiones.

\section{CONCLUSIÓN}

El sensor inercial es una herramienta práctica que permite que los entrenadores analicen la información pertinente sobre la cantidad y el ritmo de los golpes. Puede ayudarles a recomendar mejor las sesiones según los objetivos de los diferentes períodos de entrenamiento. Se garantiza que los estudios longitudinales futuros establecerán referencias sobre el número óptimo de golpes para mejorar el rendimiento, sin incrementar el riesgo de lesiones.

\section{REFERENCIAS}

Cam, I., Turhan, B. \& Onag, Z. (2013). The analysis of the last shots of the top-level tennis players in open tennis tournaments. Turkish Journal of Sport and Exercise 15(1), 54-57.

Gescheit, D.T., Cormack, S.J., Duffield, R., et al. (2017). Injury epidemiology of tennis players at the 2011-2016 Australian Open Grand Slam. Br J Sports Med, 51(17), 1289-1294. https://doi.org/10.1136/bjsports-2016-097283

Guillot, A., Genevois, C., Desliens, S., Saieb, S., \& Rogowski, I. (2012). Motor imagery and 'placebo-racket effects' in tennis serve performance. Psychology of Sport and Exercise, 13, 533- 540. https://doi.org/10.1016/j.psychsport.2012.03.002

Murphy, A.P., Duffield, R., Kellett, A., \& Reid, M. (2016). A comparison of the perceptual and technical demands of tennis training, simulated match play, and competitive tournaments. International Journal of Sports Physiology and Performance, 11(1), 40-47. https://doi.org/10.1123/ijspp.2014-0464

Myers, N.L., Sciascia, A.D., Kibler, W.B., \& Uhl, T.L. (2016). Volumebased Interval Training Program for Elite Tennis Players. Sports Health, 8(6), 536-540. https://doi.org/10.1177/1941738116657074

Perri, T., lan Norton, K.I., Bellenger, C.R., \& Murphy, A.P. (2018) Training loads in typical junior-elite tennis training and competition: implications for transition periods in a highperformance pathway, International Journal of Performance Analysis in Sport, 18(2), 327-338. https://doi.org/10.1080/24748668.2018.1475198

Pluim, B.M., \& Drew M.K. (2016). It's not the destination, it's the 'road to load' that matters: a tennis injury prevention perspective $\mathrm{Br} J$ Sports Med, 50(11), 641-642. https://doi.org/10.1136/bjsports$\underline{\text { 2016-095997 }}$
Reid, M., Cormack, S.J., Duffield, R., et al. (2018). Improving the reporting of tennis injuries: the use of time or workload data as the denominator. Br J Sports Med, 0:1-2.

Reid, M., Morgan, S., \& Whiteside, D. (2016). Matchplay characteristics of Grand Slam tennis: implications for training and conditioning. Journal of Sports Sciences, 34(19), 1-8. https://doi.org/10.1080/02640414.2016.1139161

Rogowski, I., Creveaux, T., Genevois, C., Klouche, S., Rahme, M., Hardy, P. (2016). Upper limb joint muscle/tendon injury and anthropometric adaptations in French competitive tennis players. Eur J Sport Sci, 16(4), 483-9. https://doi.org/10.1080/17461391.2015.1031712

Soligard, T., Schwellnus, M., Alonso, J.M. et al. (2016). How much is too much? (Part 1) International Olympic Committee consensus statement on load in sport and risk of injury. Br J Sports Med, 50(17), 1030-1041. https://doi.org/10.1136/bjsports-2016$\underline{096581}$ https://doi.org/10.1136/bjsports-2016-096583

Whiteside, D., Cant, O., Connoly, M., \& Reid, M. (2017). Monitoring Hitting Load in tennis using inertial sensors and machine learning. Int J Sports Physiol Perform, 12(9), 1212-1217. https://doi.org/10.1123/ijspp.2016-0683

CONTENIDO ITF ACADEMY RECOMENDADO (HAZ CLICK ABAJO)

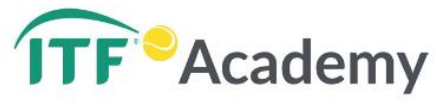

Derechos de Autor (c) 2018 Cyril Genevois, Christel Amsallem, Cédric Brandli y

Isabelle Rogowski

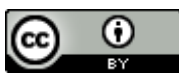

Este texto está protegido por una licencia CreativeCommons 4.0 .

\footnotetext{
Usted es libre para Compartir —copiar y redistribuir el material en cualquier medio o formato- y Adaptar el documento - remezclar, transformar y crear a partir del material- para cualquier propósito, incluso para fines comerciales, siempre que cumpla la condición de:

Atribución: Usted debe dar crédito a la obra original de manera adecuada, proporcionar un enlace a la licencia, e indicar si se han realizado cambios. Puede hacerlo en cualquier forma razonable, pero no de forma tal que sugiera que tiene el apoyo del licenciante o lo recibe por el uso que hace de la obra.
} Resumendelicencia - Textocompletodelalicencia 